\title{
Ist ihre Heimat heute noch vor Madrid? Der Spanische Bürgerkrieg und die deutschsprachige Literatur
}

Georg PICHeER

Universidad de Alcalá

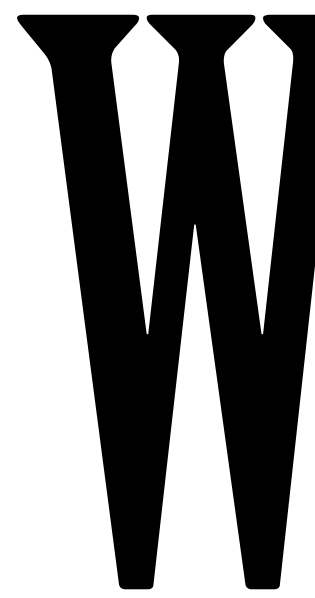

ir leben in Zeiten der Erinnerung, des kollektiven, sozialen, kulturellen Gedächtnisses, der Aufarbeitung der Vergangenheit, der Jahres- und Gedenktage. Doch innerhalb dieses Erinnerungstaumels funktioniert das Gedächtnis sehr selektiv, wird von Interessen, Konjunkturen und oft auch von einem nicht immer Gutes verheißenden Drang nach Rechtfertigung bestimmt. Verschiedene Ereignisstränge der Geschichte werden bis ins kleinste Detail beschrieben und erforscht, andere hingegen blieben brach liegen, wenn sich nicht einige Wenige ihrer annehmen würden. Die Erinnerung ist immer von aktuellen Bedürfnissen durchdrungen, der Blick auf die Vergangenheit nie von der Gegenwart unbeeinflusst und vorurteilsfrei.

Eine dieser Leerstellen im aktuellen Diskurs über die Vergangenheit ist das Erbe der Geschichte des linken Widerstands gegen die faschistischen Diktaturen in der ersten Hälfte des 20. Jahrhunderts. Nach dem Zweiten Weltkrieg vereinnahmte die kommunistische Historiographie diese Tradition für sich, freilich in einer ideologisch gereinigten, exklusiven Form. In Westeuropa begann man erst in den sechziger Jahren in größerem Ausmaß, eine nicht orthodox kommunistischeFassung dieser Tradition zu schreiben, diese Geschichte und die ihr innewohnenden Geschichten für die Gegenwart zu retten. Seit dem Fall des Eisernen Vorhangs, so scheint es nun, hat man sich anderen, politisch weniger kompromittierenden Themen zugewandt, nicht nur in der Literatur und im Kulturbetrieb, auch in der Wissenschaft. Die Vergangenheit wurde ihrer ideologischen Komponenten benommen, der Widerstand gegen alle möglichen Regimes ad acta gelegt, es war und ist immer mehr von Opfern, unschuldig Schuldigen, Mitläufern gegen ihren Willen, der menschlichen Seite, ja der Faszination der «Bösen» die Rede. Natürlich haben diese Annäherungen ihre Rechtfertigung, zeigen historische Tatsachen und Phänomene auf, die von Bedeutung sind, doch lassen sie eine Dimension außer Acht, die seit jeher eine der Triebkräfte der Geschichte war: die kathartische, vorbildhafte Funktion des Widerstands gegen jede Form von ungerechtfertigter politischer Gewalt.

Beispielhaft nachzuvollziehen ist das Phänomen an einem der «Opfer» dieser Tendenz zum Apolitischen: an der Literatur in ihren vielfältigen Formen, die in den deutschsprachigen Ländern über den Spanischen Bürgerkrieg geschrieben wurde. Bis in die frühen siebziger Jahre war sie beinahe ausschließliches Gut einer kommunistischen Historiographie und Philologie. Erst im Zuge der Auseinandersetzung mit dem Exil der dreißiger und vierziger Jahre begann vor nunmehr drei Dekaden in Westdeutschland, in Österreich und in der Schweiz eine intensivere Beschäftigung mit den Autoren, die zumeist aus autobiographischer Perspektive über ihre Erlebnisse im Spanischen Bürgerkrieg berichtet hatten. Zahlreiche Bücher, die in Exilverlagen erschienen waren, wurden neu aufgelegt, andere überhaupt zum ersten Mal veröffentlicht. Ehemalige Spanienkämpfer konnten ihre Memoiren publizieren, Anthologien mit Erinnerungen und Dokumenten wurden herausgegeben, es entstand eine Vielzahl von historischen und philologischen Arbeiten. Den Gipfelpunkt dieser Auseinandersetzung stellte der fünfzigste
Jahrestag des «Aufstands der Generäle» im Jahr 1986 dar. Danach wurde es jedoch bald wieder still, sieht man von einzelnen Ausnahmefällen ab. Dazu gehören so beispielhafte Werke wie das von Hans Landauer und Erich Hackl erstellte Lexikon der österreichischen Spanienkämpfer oder, im Bereich der Literaturwissenschaft, der Sammelband Erinnern und Erzäblen, der deutschsprachige und spanische Autoren im Zuge der «Erinnerungskultur» analysiert. Kaum nötig zu erwähnen, dass ein Großteil der Bücher über den Bürgerkrieg heute vergriffen ist; bestenfalls im Rahmen von Gesamtausgaben wurden einige Texte neu ediert, wie es etwa bei Gustav Regler der Fall war.

Anders als in den deutschsprachigen Ländern, hat der Bürgerkrieg in Spanien selbst sozial wie auch literarisch große Aktualität. Dreißig Jahre nach Francos Tod wird, von links wie von rechts, die Geschichte neu aufgerollt. Während rechte Historiker wie Pío Moa oder César Vidal in revisionistischer Absicht die alten franquistischen Thesen und Interpretationsmuster neu aufleben lassen, kommt es zum ersten Mal zu einer Auseinandersetzung mit den republikanischen Opfern, den Widerstandskämpfern des maquis und den spanischen Exilanten in Europa und Lateinamerika jenseits akademischer Kreise. Die Polemik um die Rückgabe eines Teils der im Archivo General de la Guerra Civil in Salamanca gelagerten Akten nach Katalonien, die immer breitere Aufmerksamkeit, die die Öffnung von Massengräbern aus der repressiven Zeit des Franquismus findet, und die Debatten um die von der Regierung lang geplante Ley de recuperación de la memoria histórica zeigen, dass siebzig Jahre nach dem Beginn des Bürgerkriegs und dreißig Jahre nach dem Ende der franquistischen Diktatur nun endlich eine umfas- 


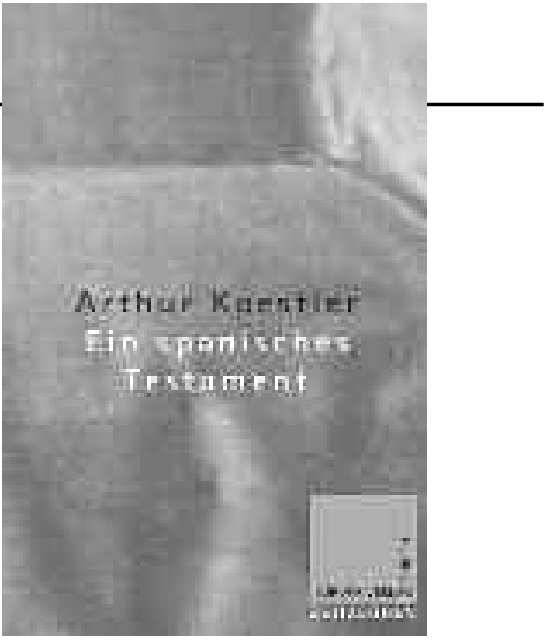

sende, intensiv und emotional betriebene "Aufarbeitung» der im Zuge der transición unterdrückten Vergangenheit eingesetzt hat. In der Literatur hat das Thema auf mehrfache Weise großes Echo gefunden. Auf der einen Seite ist eine «Bürgerkriegs- und Franquismusindustrie» entstanden, die mit Werken wie dem Edelkitschroman La voz dormida von Dulce Chacón oder dem sowohl literarisch als auch ideologisch unredlichen Soldados de Salamina eines Javier Cercas hohe Profite macht. Spätestens seit Cercas' Erfolg hat eine beinahe unüberschaubare Welle von mehr oder minder geglückten Produkten über Bürgerkrieg und Franquismus den Buchmarkt und die Kinos überschwemmt, die auf das erwachte Interesse für die eigene Vergangenheit, aber auch auf Verkaufs- und literarische Modestrategien zurückzuführen ist. Andererseits wurden wichtige zeitgenössische Romane neu aufgelegt, darunter Arturo Bareas Trilogie La forja de un rebelde oder Días de llamas von Juan Iturralde.

Aber auch in Spanien ist die - fiktionale oder autobiographische - Literatur, die von deutschsprachigen Autoren über den Bürgerkrieg geschrieben wurde, Stiefkind geblieben. Ebenso wie die Teilnahme von Deutschen, Österreichern und Schweizern in den Reihen der Internationalen Brigaden bislang kaum wahrgenommen wurde, ${ }^{1}$ sind in Spanien die meisten literarischen Texte so gut wie unbekannt geblieben. Ernest Hemingway, André Malraux, George Orwell, Georges Bernanos oder John Dos Passos zählen zu den Standardautoren über den Bürgerkrieg. Texte deutschsprachiger Autoren wurden hingegen bislang kaum übersetzt, sieht man bezeichnenderweise von Werken ab, die auf Englisch verfasst worden waren, so etwa The Spanish Cockpit des gebürtigen Wieners Franz Borkenau oder Arthur Koestlers Spanish Testament. Ein Verlust aus zwei Gründen: Historisch gesehen bringt sich Spanien um einen Teil seiner eigenen Vergangenheit, denn die autobiographischen gehören ebenso wie die fiktionalen Texte zur spanischen Geschichte. Und in literarischer Hinsicht stehen einige Romane Werken wie Hemingways For whom the bell tolls oder Malraux' L'espoir nicht viel nach.

\section{Die deutschsprachige Literatur zum Bürgerkrieg}

Der Bürgerkrieg war zu seiner Zeit ein wichtiges Thema sowohl für NS- als auch für Exil-Autoren und brachte eine umfassende literarische Produktion hervor. Für das nationalsozialistische Deutschland war der Bürgerkrieg politisch und militärisch ein Übungsfeld, auf dem die neueste Kriegstechnik erprobt werden konnte: die massive Bombardierung ganzer Städte, wie das traurige Beispiel Guernica zeigt, als die Legion Condor die symbolträchtige baskische Stadt grundlos in Schutt und Asche legte. Zugleich diente er als Beispiel für die moralische Aufrüstung der Bevölkerung, als ideologische Vorbereitung auf den Zweiten Weltkrieg: In Spanien «muss die Entscheidung fallen zwischen Bolschewismus, das heißt Zerstörung und Anarchie auf der einen, Autorität, das heißt Ordnung und Aufbau auf der anderen Seite» ${ }^{2}$, verkündete Joseph Goebbels in seiner Parteitagsrede aus dem Jahr 1937. So erschienen an die hundert Bücher zum Thema, Reportagen, Augenzeugen- und Erlebnisberichte, Propagandaschriften, aber auch einige Romane. Im Mittelpunkt stand der Einsatz der Legion Condor, zu dem allein rund zwanzig Werke veröffentlicht wurden, die jedoch erst nach 1939, also nach dem Ende des Bürgerkriegs, verlegt werden konnten. Bis dahin musste die deutsche Betei- ligung aufgrund des Nichteinmischungspaktes zwischen England, Frankreich, Italien und der Sowjetunion zumindest offiziell geheimgehalten werden, ein recht schwieriges Unterfangen, trafen denn die ersten Flugzeuge bereits Ende Juli 1936 ein und erfolgte der Abzug der Legion Condor im Mai 1939. «Stars» des nationalsozialistischen Literaturbetriebs wurden nach Spanien geschickt wie Edwin Erich Dwinger oder wie Werner Beumelburg beauftragt, die Geschichte der Legion Condor für die Nachwelt festzuhalten. Im Mittelpunkt stand dabei stets die deutsche Beteiligung am Bürgerkrieg, standen die Heldengeschichten der deutschen Flieger.

Eines der wenigen spanischen Themen, das mehrfach von nationalsozialistischen Autoren ausgeschlachtet wurde, war die Belagerung des Alcázar von Toledo zwischen Juli und September 1936, als 1800 Personen in der Festung den «bolschewistischen Horden» trotzten und schließlich von franquistischen Truppen befreit wurden. Diese mythenbefrachtete Symbolik der bedrängten «Guten», die sich militärisch straff organisiert gegen das sie umbrandende «Böse» zur Wehr setzten, passte hervorragend ins Konzept des Volkes ohne Raum, das von allen Seiten bedroht wurde und sich als «Insel im roten Meer» bewähren musste. Diese Texte dienten in erster Linie dazu, das deutsche Volk auf den heraufziehenden Weltkrieg vorzubereiten, Helden- und Kriegertum zu progagieren und die Leser auf zumeist sehr triviale Weise zu indoktrinieren. Sätze wie die folgenden sind in fast allen Büchern zu finden, wenngleich sie nicht immer mit demselben Pathos vorgebracht wurden: «Ein mächtiger Krieg, von Moskau geschürt, entbrennt, wie ihn Spanien in dieser Form noch nie im Lande erlebte. Es ist kein Bürgerkrieg, sondern ein Krieg der Anarchie und des 
Wahnsinns gegen Zivilisation und Vernunft, ein Kampf fanatischer Bosheit und unmenschlicher Leidenschaften gegen Ehre und Freiheit, ein Kampf der Gottlosigkeit gegen den Gottesglauben, ein Kampf der Gottlosenpest des Bolschewismus gegen ein christliches und nationales Spanien.» ${ }^{3}$

Da fast alle nationalsozialistischen Texte - abgesehen von ihrer historischen Bedeutung und ihrer oft unverstellt zu Tage tretenden ideologischen Pathologie-nicht sehr relevant und zudem literarisch kaum von Interesse sind, sind sie heute völlig zu Recht in Vergessenheit geraten.

\section{Exilliteratur}

Anders steht es um die Texte, die von Exilautoren geschrieben wurden. Die Bandbreite der in ihnen behandelten Themen ist weitaus größer, ihre ideologische Ausrichtung vielschichtiger und deswegen historisch ergiebiger, und ihre literarische Qualität ist ungleich besser. Dies hängt damit zusammen, dass ein Großteil der heute noch bedeutenden zeitgenössischen deutschen und österreichischen Schriftsteller ins Exil gehen musste, sei es aus rassischen, sei es aus politischen Gründen. Für viele von ihnen war der Bürgerkrieg von enormer Bedeutung, da sie hier nach den Jahren der demütigenden Vertreibung eine Möglichkeit fanden, über den Widerstand gegen den Faschismus zu schreiben und in vielen Fällen auch gegen ihn zu kämpfen. An die achtzig deutschsprachige Schriftsteller bereisten Spanien oder schlossen sich den Internationalen Brigaden an, die meisten aus einem Grund, den Gustav Regler auf den Punkt brachte: «Für mich ist die Emigration zu Ende! » ${ }^{4}$ Die Emigration war zu Ende, da man hier handeln konnte, als Schriftsteller oder Brigadist, man war kein Fremder, kein Getriebener mehr, sondern aktiv am Kampf gegen den Faschismus beteiligt. Viele andere kamen nach Spanien, um darüber zu schreiben oder die Republik propagandistisch zu unterstützen: Klaus und Erika Mann schilderten ihre Eindrükke von einer Spanienreise in einer Artikelreihe. Egon Erwin Kisch schrieb zahlreiche Reportagen vor allem über die Internationalen Brigaden. Der Lyriker Erich Arendt, der bereits seit 1934 auf Mallorca lebte, verfasste Gedichte und Prosa und meldete sich schließlich in eine spanische Einheit. Zugleich lernte er hier auch Spanisch und wurde in der DDR zu einem der

bedeutendsten Übersetzer spanischsprachiger Lyrik. Der expressionistische Anarchist Carl Einstein schrieb über seine Teilnahme in der Kolonne Durruti. Ernst Toller organisierte einen Hilfsfond für spanische Kinder und versuchte durch öffentliche Veranstaltungen in Europa und den USA Geld dafür aufzutreiben; das Scheitern dieses Hilfsfonds und die Niederlage der Republik waren nicht unwesentlich für die Depression verantwortlich, aus der heraus er am 22. Mai 1939 in New York Selbstmord beging, drei Tage nach Francos Siegesparade in Madrid. Spätere DDR-Autoren wie die Schriftsteller Rudolf Leonhard, Erich Weinert oder Hans Marchwitza lasen aus ihren Werken in Erholungsheimen für verwundete Interbrigadisten, der Sänger Ernst Busch unternahm eine gut dokumentierte Tournee durch Spanien und gab Konzerte in Krankenhäusern und an der Front. Anna Seghers, Ludwig Renn, Bodo Uhse, Kisch, Regler, Marchwitza, Weinert und andere nahmen am wohl größten literarischen Ereignis teil, das während des Bürgerkriegs in Spanien stattfand: am II. Internationalen Schriftstellerkongress, der im Juli 1937 in Valencia und Madrid tagte und eine Fortsetzung in Paris fand.

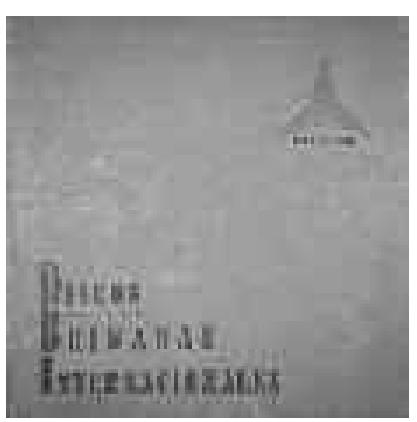

Die Mappe enthält drei Schellacks mit insgeDemtsechs Liedern. ErnstBuschnahm siewähischen Bürgerkriegs 1937 im Odeon-Studio in Barcelona auf. Er publizierte die Lieder der Internationalen Brigaden darüber hinaus auch in Buchform. nisberichte, Erzählungen und Gedichte bis hin zu Memorien, die erst Jahrzehnte später veröffentlicht werden sollten. Die Mitglieder der Internationalen Brigaden wurden von eigenen «Kulturarbeitern» angehalten, ihre Erlebnisse niederzuschreiben, die dann in Bataillonszeitungen und anderen Sammlungen veröffentlicht wurden. Das Ziel war, auf diese Weise eine kollektive Geschichte «von unten» zu schreiben, aus der Sicht jener Akteure, die bis dahin stets still geblieben waren; und dies lange bevor die Historiographie sich in den achtziger Jahren dieser Methode der Oral history besann, um das Alltagsleben anhand ihrer Protagonisten festzuhalten. Das Resultat dieser gemeinschaftlichen Anstrengungen waren verschiedene Bände, die teils noch zu Zeiten des Bürgerkriegs, teils später erschienen. Literarisch sind sie nur selten relevant, doch geben sie perspektivenreich und vielschichtig Auskunft über das Leben der unterschiedlichen deutschsprachigen Brigaden. Bereits 1938 erschien in einem spanischen Verlag die von Alfred Kantorowicz herausgegebene Sammlung Tschapajew. Das Bataillon der 21 Nationen, in dem das Schicksal dieses multinationalen, zur XIII. Brigade gehörenden Bataillons von

\section{Dokumentarliteratur}

Da die meisten Autoren in Kontakt mit den Internationalen Brigaden standen oder ihnen angehörten, verwundert es nicht, dass nur sehr wenige Bücher Aspekte behandeln, die nicht unmittelbar mit den Kämpfen zu tun hatten. Dazu zählen vor allem die Spanienbücher von Peter Merin (Spanien zwischen Tod und Geburt) und Franz Borkenau (The Spanish Cockpit), die einen weiten Horizont aufweisen und die politischen, sozialen und kulturellen Bedingungen des Bürgerkrieg zu analysieren versuchen. Wegen ihrer objektiven Betrachtungsweise, ihres Detailsreichtums und ihrer Fülle an Informationen sind sie auch heute noch von Interesse.

Die Zahl der Texte von Mitgliedern der Internationalen Brigaden ist Legion. Sie reicht von Tagebüchern über kurze Erleb- seinen Mitgliedern nacherzählt wird. In seiner Mischung aus einer auf spätere Erinnerung bedachten Zeugenschaft, politischer Propaganda, verschiedenen Gattungen und vielstimmigen Schreiben ist das Buch ein wertvolles Dokument, das 1956 in der DDR ohne Kürzungen und Auslassungen inzwischen unliebsam gewordener Mitkämpfer wieder aufgelegt wurde. Im Gefolge von Kantorowicz' Buch erschienen andere Texte, die ebenso versuchten, den Bürgerkrieg aus der Perspektive der Mitkämpfer zu schildern, etwa die von Willi Bredel in Spanien begonnene, aber erst 1977 in der DDR herausgegebene, weitaus linientreuere "Geschichte der XI. Internationalen Brigade» mit dem Titel Spanienkrieg. Darin wurden zu jenem Zeitpunkt für Tabu erklärte Autoren ausgespart, die Ausrichtung des Buches entspricht voll und ganz der offiziellen Sichtweise. 
Für Spaniens Freiheit. Österreicher an der Seite der Spanischen Republik (1986) stellt hingegen in ideologischer Bandbreite und unter Zuhilfenahme von Augenzeugenberichten, Gesprächen, Tagebucheintragungen, Ausschnitten aus Memoiren die Beteiligung der Österreicher am Spanischen Bürgerkrieg dar.

Andere Anthologien wie etwa Der Spanische Bürgerkrieg in Augenzeugenberichten (1967) oder die zum fünfzigsten Jahrestag erschienenen Geschichten aus der Geschichte des Spanischen Bürgerkriegs versuchen, in Abgrenzung von der Vereinnahmung durch die parteikommunistische Sichtweise, ein ausgewogenes Bild des Bürgerkriegs zu zeichnen, aber auch Texte zugänglich zu machen, die bis dahin verstreut veröffentlicht worden waren. Bis heute gibt es aber immer noch keine wirklich groß angelegte Sammlung, die Spreu von Weizen trennen und die auch gegenwärtig noch interessanten autobiographischen oder dokumentarischen Texte zugänglich machen würde.

Eine andere Quelle, die an der Grenze zwischen Historiographie und Literatur steht, sind die Memoiren zahlreicher Spanienkriegsteilnehmer, die zum Teil mit erheblicher Verspätung, manchmal auch erst nach dem Tod des Autors erschienen. Eine der ersten Autobiographien war die des österreichischen Kommunisten Sepp Plieseis, der in Vom Ebro zum Dachstein (1946) seinen Weg von Österreich über Spanien in die Sowjetunion und zurück nach Österreich nachvollzieht. Willy Brandt schilderte in Draußen (1967) seine Reise im Auftrag der Sozialistischen Jugend durch Spanien, der österreichische Sozialdemokrat und Weltkriegsgeneral Julius Deutsch (Ein weiter Weg, 1960) beschrieb seine militärischen Funktionen im Dienste der Republik, bei denen er unter anderem mit der Verteidigung der Mittelmeerküste beauftragt war. Relativ spät und zumeist aus Anlass eines Jahrestages des Bürgerkriegs kamen die Autobiographien von Josef Schneeweiß (Keine Führer, keine Götter, 1986), Walter Fischer (Kurze Geschichten aus einem langen Leben, 1986) oder von Hans Hutter (Spanien im Herzen, 1996) heraus. Mit literarisch weiter gespannten Ambitionen schrieb sich der Schriftsteller Albert Vigoleis Thelen in Die Insel des zweiten Gesichts (1953) in die sehr spanische Tradition des Schelmenromans ein und zeichnete ein humorvolles Bild seines Exils auf
Mallorca, das zu Beginn des Bürgerkriegs abrupt endete. Die drei ausführlichsten autobiographischen Zeugnisse sind die Spanienbücher von Alfred Kantorowicz, Ludwig Renn und Theodor Balk.

Alfred Kantorowicz kann als der pointierteste Chronist der Internationalen Brigaden gelten. Als Offizier in unterschiedlichen Funktionen tätig, hatte er Einblick in alle Ebenen der Brigaden und hielt vom Dezember 1936 bis zu seiner Verwundung im Juli 1937 eine Unmenge von Ereignissen fest, schrieb sowohl über den Alltag der Kämpfe, seine Tätigkeit in der Organisation, seine «Kulturarbeit» und seine unzähligen Begegnungen mit führenden Persönlichkeiten der spanischen Republik und den zahlreichen Ausländern, die sich im Land aufhielten. Vor allem in Hinblick auf die deutsche Teilnahme kann sein Buch als detailreicheQuelle gelten, da der umtriebige, mitunter auch etwas geschwätzige und eilfertige Kantorowicz überall zugleich sein wollte und es oft auch zu sein wusste. Kaum ein anderer hat den Alltag derart umfassend und vielschichtig wiedergeben. Sein Spanisches Kriegstagebuch erschien erstmals 1948 in der DDR und in seiner endgültigen Form 1979, lange nach dem Übertritt des Autors in die BRD.

Der spanische Krieg

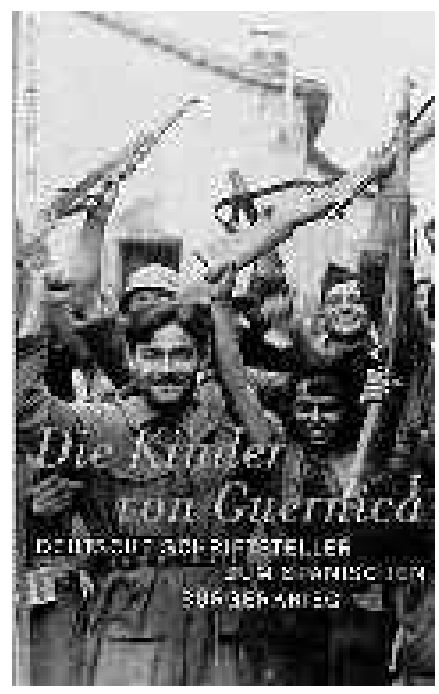

preußischem Drill auszurichten. Sein Buch ist vor allem hinsichtlich der militärtechnischen Details und der Funktionsweise der Brigade interessant, wenngleich die politische Engstirnigkeit und auch die Eitelkeit des Autors seine historische Bedeutung schmälert. Zugleich ist es eines der Beispiele für die manchmal doch recht deutliche «interkulturelle Inkompetenz» einiger Internationaler, die nicht in der Lage oder willens waren, ihren deutschen Blick für die spanische Wirklichkeit zu öffnen und vor allem sich selbst in den Mittelpunkt stellten: so etwa, wenn Renn die Tortilla etwas verschroben als «einen Eierkuchen mithineingebackenen Kartoffeln» beschreibt oder Aussagen trifft wie «der gewöhliche Spanier ist ja harmlos und ehrlich» ${ }^{5}$.

Ein eigenartiges Schicksal hat der sehr spät, nämlich erst 1996 erschienene autobiographische «Tagebuch-Roman» Wen die Kugel vor Madrid nicht traf von Theodor Balk. Balk hatte seine Tagebuchnotizen aus dem Bürgerkrieg, die vom Februar 1937 bis Februar 1939 reichen, im Exilversteckt und verloren, bis er sie unerwarteterweise ein Vierteljahrhundertspäter wiedererhielt. So verband er seine sporadischen, unter dem Eindruck des unmittelbar Erlebten entstandenen Aufzeichnungen mit von Ludwig Renn ist literarisch geformter als Kantorowicz' Tagebuch, seine politische Ausrichtung nimmt ihm jedoch einiges an Wert. Ideologisch ist das 1955 erschienene Buch von allem gereinigt, was in der spätstalinistischen Ära unerwünschtwar. Renn, einer der Heroen der deutschsprachigen Internationalen, gibt bei aller Liebe zum Detail und zum spanischen Umfeld eine doktrinär kommunistische Version des Bürgerkriegs wieder: Häufig attackiert er die anderen politischen Positionen, Parteien und Politiker, und er eliminiert alle Renegaten aus seinen Erinnerungen. Der Adelige Renn, der mit richtigem Namen Arnold Vieth von Golßenau hieß, wurde als ehemaliger Weltkriegsoffizier und militärischer Fachmann Stabschef der XI. Internationalen Brigade und versuchte, sie nach erklärenden, überleitenden Zwischentexten, die er aus der Erinnerung schrieb. Das Ergebnis ist ein Hybrid, in dem das Direkte, Lückenhafte und Spontane der Aufzeichnungen mit dem reflektierenden Wissen um die historischen Ereignisse danach verbunden ist. Balk, der Arzt der XIV. Brigade war, ist weniger als Kantorowicz und Renn bemüht, sich selbst in den Mittelpunkt zu stellen, seine Notizen sind bescheidener im Anspruch, das Bild, das er vom Alltagsleben in seiner Einheit zeichnet, ist unprätentiöser, in historischer Hinsicht und als Dokument über die Internationalen Brigaden wohl auch weniger aufschlussreich. Doch wird dieses Manko durch die informationsreichen nachträglichen Ausführungen wiedergutgemacht, die Balk aus einer melancholischen Distanz heraus verfasste. 


\section{Die literarische Verarbeitung}

Ein Charakteristikum der Bürgerkriegsliteratur klingt im Untertitel von Balks autobiographischem «Tagebuch-Roman» an: das Ineinander von Realität und Fiktion. Die Grenze zwischen fiktionalen und nicht fiktionalen Elementen ist in vielen Texten fließend. Nicht fiktionale Texte wie Augenzeugenberichte, Tagebücher, Erinnerungen, Reportagen oder Zeitungsartikel wurden von den Autoren oft mit literarischen Techniken gestaltet; fiktionale Texte dienten wiederum der Auseinandersetzung mit dem realen Geschehen, waren ein Mittel, über den Bürgerkrieg zu schreiben und reale politische oder ideologische Probleme abzuhandeln. Eine strikte Trennung ist somit für die Bürgerkriegsliteratur eher belanglos, da die verschiedenen literarischen Ausdrucksformen sich um ein reales historisches Ereignis gruppierten, und wenn sie nicht den Anspruch der historischen Wahrheit hatten, so doch den der Wahrhaftigkeit. Zu messen sind diese Texte wohl nur an ihrer Bemühung um Aufrichtigkeit bei der Wiedergabe der Geschehnisse und an ihrer Unvoreingenommenheit hinsichtlich der widerstreitenden ideologischen Positionen. Werke, die sich des Ereignisses bedienen, um «allgemein menschliche Probleme» darzustellen, sind eher die Ausnahme und wurden von Autoren verfasst, die nicht am Bürgerkrieg teilnahmen.

Insofern sind die vier Interbrigadenromane, die von deutschen Autoren geschrieben wurden, allesamt autobiographischen Ursprungs, auch wenn sie mehr oder weniger deutlich reale Geschehnisse schildern und von realen, oft nur leicht literarisierten Personen erzählen. Gustav Reglers Der große Kreuzzug, berichtet aus verschiedenen Perspektiven über die Zeit zwischen November 1936 und Juni 1937, eben die Zeit, in der Regler selbst als politischer Kommissar der XII. Brigade tätig war. Wie in allen Romanen über die Brigaden hat Der große Kreuzzug keinen «roten Faden», sondern gestaltet vornehmlich die kriegerischen Auseinandersetzungen in ihrer chronologischen Abfolge nach, aber auch den Zustand im Hinterland und in $\mathrm{Ma}$ drid oder die politischen Umstände und Diskussionen in den großteils kommunistischen Einheiten. Durch seine vielperspektivische Erzählweise, die neben den beiden Protagonisten - Albert, der Kommissar, in dem leicht der Autor selbst zu erkennen ist, sowie Werner, der Chefarzt des Bataillons - eine Unzahl anderer Figuren in Innen- und Außensicht darstellt, zeichnet Regler ein umfassendes, detailreiches und reflektiertes Bild des Lebens in den Internationalen Brigaden. Seine genaue Kenntnis der politischen Hintergründe kam Regler dabei zugute, denn er zählte zu den prominentesten deutschen Internationalen in Spanien. Er war in Kontakt mit beinahe allen wichtigen Persönlichkeiten, für seinen Optimismus und seine Energie bekannt und geschätzt, sein persönlicher Geltungsdrang brachte ihm den Ruf ein, der meistfotografierte Brigadist zu sein. ${ }^{6}$ Der Roman ist zugleich ein Beispiel für die Schwierigkeiten, Texte über den Bürgerkrieg zu publizieren: Er erschien 1940 in US-amerikanischer Übersetzung mit einem Vorwort von Ernest Hemingway, auf Deutsch kam er als Das große Beispiel erst 1976 und in einer endgültigen Version 1996 unter dem definitiven Titel und versehen mit den Tagebüchern aus seiner Zeit in Spanien heraus. In dem Roman werden noch vorsichtig die Bedenken zum Ausdruck gebracht, aufgrund derer Regler später die KP verließ und zu einem der bekanntesten Renegaten wurde. Weitaus deutlicher schrieb Regler über seinen politischen Wandel im Spanienkapitel sei-

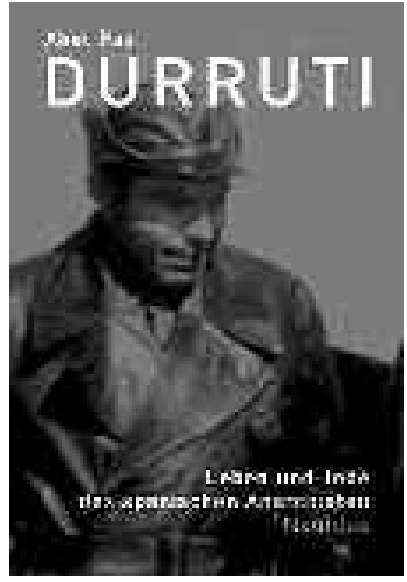

glied des «Bundes proletarisch-revolutionärer Schriftsteller» bekannt geworden war, erzählt in Begegnung am Ebro (1939) seine Erlebnisse im Thälmann-Bataillon der XI. Brigade. Nicht nur ideologisch ist Bredels Text platter und eindeutiger als der Reglers, der Roman an sich gibt ein GutBöse-Schema wieder, das bis in die Physionomie der Figuren reicht: Politisch zweifelhafte Figuren sind immer auch hässlich. In Begegnung am Ebro findet sich eine Reihe von Themen, die in vielen Texten über die Internationealen Brigaden wiederkehren: über den Zwiespalt der Schriftsteller, sich zwischen "Schwert und Feder» entscheiden zu müssen, über die Angst vor den Kämpfen, über Verrat und Spionage in den eigenen Reihen, über das Gefühl, das wahre Deutschland zu vertreten, und die Notwendigkeit, die Verbrechen des Dritten Reiches im Kampf gegen den Faschismus wiedergutzumachen. Bredel rückt auch, mehr als es andere Autoren tun, Spanier in den Mittelpunkt, an denen er das politische Erwachen durch die Auseinandersetzung mit den internationalen Freiwilligen zeigt. Freilich tut er dies ganz im Sinn der kommunistischen Sichtweise, was seinem Roman nicht immer zum Besten gereicht.

Eine andere Facette, die in allen Texten zumindest ner Lebensgeschichte Das Ohr des Malchus (1958). In ihr nahm er sich nach seinem Bruch mit den Kommunisten, der sich im französischen Internierungslager Le Vernet und in Mexiko vollzog, kein Blatt mehr vor den Mund und kritisierte deren Politik in Spanien, die er, wie viele andere auch, in André Marty, dem Abgesandten der Komintern, verkörpert sah.

Weitaus weniger ideologischen Schwankungen ausgesetzt waren die Autoren der anderen drei Interbrigadistenromane, ebenso wie Regler von der KP eingesetzte Politkommissare. Willi Bredel, Eduard Claudius und Bodo Uhse hielten sich relativ eng an die kommunistische Sichtweise und gelangten als Bürgerkriegsveteranen und Kulturfunktionäre in der DDR zu hohem Ansehen. Willi Bredel, der in den zwanziger Jahren als Arbeiterschriftsteller und Mit- implizit angelegt ist, tritt bei Eduard Claudius' Roman Grüne Oliven und nackte Berge (1944) deutlich hervor, nämlich die des politischen Entwicklungsromans. In ihm beschreibt der Autor die "politische Mannwerdung» des Protagonisten, hinter dem kaum verborgen Claudius selbst steht, der sich den Internationalen Brigaden anschließt und durch die Kämpfe und ideologischen Diskussionen zur Reife gelangt. Aufgrund dieser Konzentration auf eine Figur ist das historische Panorama, das der Roman bietet, denn auch eher beschränkt: Kämpfe werden ausführlich und immer wieder in sehr hartem Ton nachgezeichnet, es geht um Tod und das Töten, um Disziplin und um Soldatentum, an manchen Stellen sind die Grenzen zu rechter Landserliteratur und ihrer Betonung der Männlichkeit, ihrer absoluten 
Verdammung des «Feindes» und ihrer Misogynie nicht immer eindeutig erkennbar. Auch wenn das Ringen des Helden um politische Erkenntnis ehrlich dargestellt wird, ist es derart von bis in die Sprache reichenden ideologischen Präfigurationen gekennzeichnet, dass Claudius' Text trotz einiger beeindruckender Stellen - die Landung der Brigadisten im Hafen von Valencia etwa - wohl als der unergiebigste aller Brigaderomane gelten kann.

Den Erkenntnisgewinn eines nationalsozialistischen Offiziers hat hingegen Bodo Uhse in den Mittelpunkt seines Romans Leutnant Bertram (1943) gestellt. Nicht ohne Grund, war doch Uhse selbst bis 1930 Mitglied rechtsradikaler Organisationen und der NSDAP, bis er sich der Bauernbewegung der KPD anschloss. Der erste Teil von Uhses Roman spielt im nationalsozialistischen Deutschland, der zweite in Spanien, wo die beiden Protagonisten - der NS-Fliegerleutnant Bertram und der kommunistische Arbeiter Hein - aufeinandertreffen. Uhse kann so, als einer der wenigen deutschsprachigen Autoren, den Bürgerkrieg aus zwei Perspektiven zeigen, wenngleich er natürlich dezidiert für die republikanische Stellung nimmt. Diese im Grund sehr vielversprechende Anlage wird zum Teil dadurch unterlaufen, dass Uhse die Figuren oft sehr simpel durch seine ideologische Brille zeigt. Aus dem beabsichtigten politischen Bildungsroman wird so streckenweise ein ideologisch beeinträchtigter Thesenroman, in dem die Seite der Nationalsozialisten weitaus eindrücklicher, lebendiger und psychologisch aufschlussreicher dargestellt wird als die allzu oft auf Schemen reduzierten Interbrigadisten. Wie auch die anderen Romane hat Leutnant Bertram keine Fabel im herkömmlichen Sinn, sondern breitet in vielen nebeneinander stehenden Szenen gleichsam ein breit angelegtes Fresko vor dem Leser aus. Dieses Erzählen von lose aneinander gereihten Fragmenten, in die nur ansatzweise zeitgeschichtliche Elemente und eine weiter ausholende Reflexion über die Bedeutung des Bürgerkriegs in einem größeren Zusammenhang eingestreut sind, entsprach sicher den konkreten Eindrücken der Autoren, die an den Kämpfen der Internationalen Brigaden teilnahmen und von ihnen berichteten. Ihr $\mathrm{Zu}$ gang war die literarische Verarbeitung realen Geschehens, die eigenen Erlebnisse standen dabei immer im Zentrum und sind oft kaum verbrämt dargestellt.

Anders war der Zugang von zeitgenössischen Autoren, wenn sie nicht über die Internationalen Brigaden schrieben. Für sie standen die kriegerischen Auseinandersetzungen nicht im Mittelpunkt, sie schrieben keine linken Kriegsromane, sondern versuchten, aus den historischen Tatsachen Literatur zu machen.

Der vormals expressionistische Lyriker Karl Otten etwa war bereits 1933 nach Spanien emigriert und lebte in Cala Ratjada auf Mallorca. Dort wurde er Zeuge des Ausbruchs des Bürgerkriegs, kam kurz darauf in Haft, konnte sich aber bald nach England in Sicherheit bringen. Sein Roman Torquemadas Schatten, der ursprünglich 1938 in den Niederlanden erschienen war, zu der Zeit aber kaum Leser fand, wurde erst 1980 in Deutschland wieder aufgelegt. Otten schildert darin die Machtergreifung der Franquisten auf der Insel in den ersten vier Wochen nach dem Putsch der Generäle anhand einer Gruppe von Hauptfiguren und eines umfangreichen Ensembles an Nebenfiguren. Und er tut dies mit sehr viel Liebe fürs Detail, mit Orts- und Sachkenntnis und großer Sympathie für die Inselbewohner, vor allem für die von den Aufständischen Verfolgten. Eher als historisches Dokument will Torque-

madas Schatten Literatur sein: Der Autor verwendet zwar zahlreiche reale Elemente, verarbeitet sie aber in eine fiktionale Fabel, die er in einer hohen Sprache beschreibt, ein deutliches Echo seiner expressionistischen Vergangenheit. Zudem stellt er den Bürgerkrieg in einen größeren historischen Zusammenhang, wenn er bereits im Titel Anklänge an den berüchtigsten spanischen Großinquisitor aufkommen lässt, wobei er immer wieder das reiche, reaktionäre, katholische vom armen und fortschrittlichen Spanien unterscheidet. Dabei weist er weitaus größere psychologische Einfühlsamkeit in seine Figuren nach, gestaltet sie plastischer und tiefgründiger als andere, eher politisch ausge- richtete Autoren, die über sich selbst und ihren Kampf gegen den Faschismus berichteten. Und auch sein Blick auf Spanien ist interessierter, sachkundiger und einfühlsamer als der anderer zeitgenössischer $\mathrm{Au}$ toren - vielleicht auch deshalb, da er zwar Partei ergreift, sich aber nicht von vorgegebenen politischen Formeln beeinflussen lässt.

Sachkundig, wenngleich in anderer Hinsicht, ist auch Gustavs Reglers zweiter Bürgerkriegsroman, Juanita, 1940 in Mexiko fertiggestellt und erst 46 Jahre später erschienen. Im Gegensatz zu seinen bereits erwähnten autobiographisch inspirierten Texten ist Juanita die erfundene Geschichte einer naiven, politisch unschuldigen jungen Frau, die im belagerten Madrid zwischen die Fronten der Ideologien gerät. Regler benützt das Handlungsgerüst, um seine eigenen Zweifel am Kommunismus zum Ausdruck zu bringen, aber auch ein Bild des Bürgerkriegs zu zeichnen, das in seinem ersten Roman ausgespart blieb. Denn Juanita erzählt die unglücklicheLiebesgeschichte zwischen der Titelheldin und einem Falangisten, der wohl nicht zufälligerweise Francisco heißt, und gibt Regler Gelegenheit, ausführlich und detailreich das Leben in Madrid zu zeigen, das von internationalen Geheimagenten, Spitzeln, Politikern, Diplomaten, Journalisten und Mitglie-

dern der Quinta Columna bestimmt wird. Juanita ist zwar in seinen Intentionen, die innere Front der spanischen Republik weit ausholend, fiktional und in epischer Breite $\mathrm{zu}$ beschreiben, viel ambitionierter als Der große Kreuzzug, doch steht der Roman in literarischer Hinsicht und als zeitgeschichtliches Dokument hinter den autobiographischen Texten Reglers zurück.

Ebenso ein spanisches Schicksal hat der Roman Die Kinder von Gernika von Hermann Kesten zum Thema. Wie bereits im Titel anklingt, geht es um eine baskische Familie, die durch den Bürgerkrieg und die Bombardierung der Stadt Guernica durch die Legion Condor im April 1937 zerrissen wird. Kesten zeigt anhand des Schick- 
sals des Protagonisten Carlos, Sohn des Apothekers der Stadt, wie die politischen Ereignisse die Gesellschaft in Gute und Böse teilen. Das Böse gewinnt, die Familie muss ins Exil nach Frankreich und zerbricht nicht nur am Politischen, sondern auch an den ihr selbst innewohnenden Spannungen. Kestens Roman ist historisch und geographisch sehr genau recherchiert und beschreibt beeindruckend die Bombardierung der Stadt, die auch heute noch - und nicht nur dank Picassos Bild - als Symbol für die Brutalität und Sinnlosigkeit des Krieges gilt. Doch leidet Die Kinder von Gernika unter Kestens moralischem Manichäismus ebenso wie unter seinem Versuch, dem Text ein Lokalkolorit zu verleihen, das manchmal eher an deutsche Kleinstädte erinnert. Authentischer wirkt der zweite Teil des Textes, in dem die Familie im Pariser Exil lebt: Dies kannte der Autor aus eigener Erfahrung, so dass er hier die Probleme seiner Figuren weitaus treffender zu schildern wusste.

Die meisten ernst zu nehmenden Texte über den Bürgerkrieg wurden von linken Autoren veröffentlicht, die das Thema nutzten, um politisch, ideologisch oder moralisch gegen den Europa überziehenden Faschismus zu schreiben. Ihre Texte sind immer auch im Zusammenhang des Exils und des po-
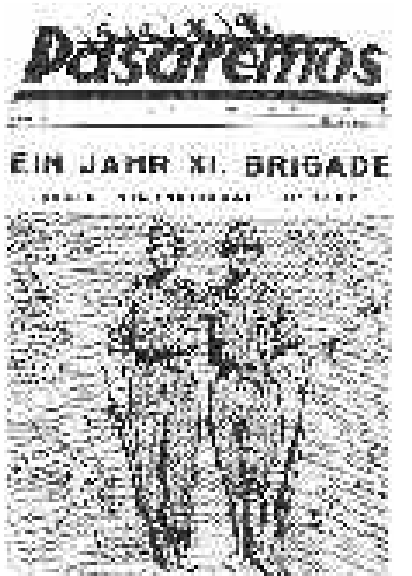
litischen Kampfes - ob mit Worten oder Taten - zu sehen. Im Gegensatz dazu nehmen die zwei Texte, die von einer katholischen Warte aus geschrieben wurden, die historischen Ereignisse nur als Erzählvorwurf, um an ihnen existenzielle Probleme abzuhandeln. Die politisch-militärische Auseinandersetzung ist bei ihnen zweitrangig, sie stellt nur einen Extremfall von menschlicher Ausgesetztheit dar, die Anlass zur Klärung anderer Fragen gibt.

Dies ist der Fall in Stefan Andres Novelle Wir sind Utopia (1942), die bezeichnenderweise im Nachkriegsdeutschland des Kalten Krieges zum Bestseller der deutschsprachigen Bürgerkriegsliteratur avancierte, und in Imma von Bodmershofs weitaus unbekannterem Roman Die Bartabnahme (1966). Da in ihnen der Bürgerkrieg keine wesentliche Rolle spielt, seien sie hier nur am Rande erwähnt.

\section{Die Gegenwart der Geschichte}

Nach dem Ende des Zweiten Weltkriegs zeigte sich, dass der Bürgerkrieg im deutschsprachigen Raum kein bedeutendes Thema mehr war, das literarisch abgehandelt werden musste. Zwar tauchte er immer wieder in Romanen und Erzählungen von «nachgeborenen» DDR-Autoren als Randthema auf, doch machten ihn nur wenige Schriftsteller, die nicht selbst an ihm teilnahmen, zum Mittelpunkt ihrer Werke, Werke die literarisch und inhaltlich nicht immer interessant waren? ${ }^{7}$. Deswegen sei hier nur auf drei Autoren eingegangen, die wesentlich neue Aspekte einbrachten.

1975 erschien der erste Band von Peter Weiss' Trilogie Die Ästhetik des Widerstands, dessen zweites Buch versucht, aus einer «erinnerungskulturellen» Position heraus die historischen Ereignisse und ideologischen Spannungen nachzuzeichnen. Weiss schickt seinen fiktiven Ich-Erzähler nach Spanien, wo er auf bekannte historische Figuren der deutschen Emigration trifft. Bedeutsam ist an Peter Weiss' genauestens recherchiertem Roman seine erzähltheoretische Position: Er kann nicht mehr ungebrochen erzählen, wie es die meisten Exilautoren bis dahin taten, sondern er bricht seinen Text dadurch, dass er ihn zu einer Reproduktion der Ereignisse gestaltet. Sein Erzähler ist kein aktiver Teilnehmer, sondern ein Medium, das dem Leser die historischen Geschehnisse und die politischen Debatten unter den Interbrigadisten vermittelt. Und Weiss nimmt in seinen Text ausführliche Beschreibungen von Werken der bildenden Kunst auf, die einerseits die dargestellten Ereignisse in einen größeren $\mathrm{Zu}$ sammenhang bringen, andererseits darauf verweisen, dass auch der Autor seine Geschichte nicht anhand der "Wirklichkeit», sondern anhand ihrer Reproduktionen in Form von Aufzeichnungen der Teilnehmer und historischen Werken gestaltet. Weiss macht so in der Gestaltung seine Arbeit offensichtlich: Indem er in der Ästhetik des Widerstands einen fiktiven Gang durch die Geschichte der Emigration und des Widerstands gegen das NS-Regime tut, versucht er, dem Leser seine Sicht dieser Ge- schichte nahezubringen: er reduziert die Geschichte auf ihre Grundzüge, so dass sie Sinn erhält.

Das Gegenteil versuchte zwei Jahre später Hans Magnus Enzensberger in Der kurze Sommer der Anarchie, dem er den provokativen Untertitel Roman gab. Enzensberger erzählt darin die Biographie des leonesisch-katalanischen Anarchisten Buenaventura Durruti nach, indem er eine Fülle von sich widersprechenden Zeugnissen nebeneinander stellt und so ein Bild vermittelt, das seine Wahrheit eben dadurch erhält, dass es jede definitive Wahrheit von vornherein leugnet. Wie Weiss geht es Enzensberger darum, historische Ereignisse wiederzugeben, zugleich aber auch seine Methode durchsichtig zu machen. So wählt und ordnet er das dokumentarische Material nach chronologischen Kriterien und gestaltet vielstimmig das Leben Durrutis, der im November 1936 in Madrid fiel. Enzensbergers eigener Beitrag sind neben der Auswahl und Ordnung des Materials einzig acht Glossen, in denen er historische, politische und ideologische Hintergründe kommentiert. Das Buch geht nur gegen Ende auf den Bürgerkrieg ein, zeigt aber an der Biographie Durrutis dessen Entstehungsbedingungen auf und entwirft ein vielschichtiges und widersprüchliches Panorma Spaniens im ersten Drittel des 20. Jahrhunderts.

Der deutschsprachige Gegenwartsautor, der am umfassendsten über Spanien und den Bürgerkrieg geschrieben hat, ist Erich Hackl. Bei ihm stehen weniger an konkreten historischen Beispielen angewandte erkenntnistheoretische Positionen wie bei Enzensberger oder die Einbettung des Ereignisses in einen politischen Gesamtzusammenhang wie bei Weiss im Vordergrund, sondern das Erinnern an die Menschen, die im Bürgerkrieg kämpften: Hackl versucht in seinen Texten, all diejenigen vor dem Vergessenwerden zu bewahren, die in ihrem Aufbegehren gegen das Unrecht von der Geschichte übergangen wurden, betreibt also im wahrsten Sinn des Wortes «Gedächtnisarbeit». Für Hackl ist die Geschichte nicht abgeschlossen, sondern wirkt in die Gegenwart herein. Bereits in seinem ersten Buch, Auroras Anlaß (1987), sind die Zweite Republik und der Bürgerkrieg präsent. In ihm berichtet er die Geschichte der Spanierin Aurora Rodríguez, die ihre Tochter zu einer Frau ohne Falsch und Makel erziehen will und 
daran scheitert. In zwei weiteren Texten, Entwurf einer Liebe auf den ersten Blick (1999) und Die Hochzeit von Auschwitz (2002), erzählt er das Leben von österreichischen Interbrigadisten nach, die sich in Spanien verliebten, heirateten und in nationalsozialistischen Konzentrationslagern umkamen. Hackl geht es dabei um die literarische Gestaltung der historischen Fakten, um Treue nicht einer abstrakten Wahrheit, sondern den realen Personen gegenüber, über die er schreibt und denen er sich verpflichtet fühlt. Und er geht immer von den historischen Vorgaben aus, die für die erzählerische Arbeit bestimmend werden: Trotz der ähnlichen Geschichten sind beide Bücher völlig unterschiedlich geschrieben, da sich die Methode des Erzählens nicht wiederholen kann, ohne zum Klischee zu werden. Hackls Material sind biographische Daten in allen möglichen Formen, Akten, Interviews, die er, nicht nur in seinen Büchern, sondern auch in vielen kleineren Porträts von Bürgerkriegsteilnehmern, literarisch formt, wobei er sich gegen jede Fiktionalisierung seines Stoffes verwahrt. Allein die Erzählweise macht die Literarizität des Textes aus.

Von allen hier kurz besprochenenen Büchern sind nur die Werke der letzten drei genannten Autoren ins Spanische übersetzt; eine spanische Übersetzung von Hermann Kestens Roman erschien 1945 in Buenos Aires, wurde aber nie in Spanien herausgegeben - ein mehr als deutliches Zeichen für das mangelnde Interesse des spanischen Verlagswesens an Büchern und Autoren, die zur Geschichte ihrer eigenen Kultur gehören. Der Bürgerkrieg ist Geschichte geworden, aber die Geschichten, die deutschsprachige Autoren über ihn geschrieben haben, scheinen für die Gegenwart nicht allzu bedeutend. "Wir im fernen Vaterland geboren,/Nahmen nichts als Hass im Herzen mit,/Aber wir haben die Heimat nicht verloren,/Unsere Heimat ist heute vor Madrid», heißt es im Lied der Internationalen Brigaden von Erich Weinert. Ob dem heute auch noch so ist und ob die Heimat der Interbrigadisten immer noch vor Madrid ist, scheint angesichts des geringen Interesses an ihnen nicht nur in Spanien wohl mehr als fraglich.

\section{Auswahlbibliographie:}

Balk, Theodor: Wen die Kugel vor Madrid nicht traf. Tagebuch-Roman über den spanischen Bürgerkrieg und das Los der Spanienkämpfer. St. Ingbert: Röhrig 1996.

Bannasch, Bettina, Christiane Holm (Hrsg.): Erinnern und Erzählen. Der Spanische Bürgerkrieg in der deutschen und spanischen Literatur und in den Bildmedien. Tübingen: Narr 2005.

Borkenau, Franz: Kampfplatz Spanien. Politische und soziale Konflikte im Spanischen Bürgerkrieg. Ein Augenzeugenbericht. Aus dem Engl. von Werner Steinbeiß. Stuttgart: Klett-Cotta 1986. (EA The Spanish Cockpit, 1938)

Bredel, Willi: Spanienkrieg. 2 Bde. Hrsg. von Manfred Hahn. Berlin, Weimar: Aufbau 1977.

Claudius, Eduard: Grüne Oliven und nackte Berge. Roman. Halle/Saale: Mitteldeutscher Verlag 1986. (EA 1944)

Enzensberger, Hans Magnus: Der kurze Sommer der Anarchie. Buenaventura Durrutis Leben und Tod. Roman. Frankfurt/Main: Suhrkamp 1972.

Für Spaniens Freiheit. Österreicher an der Seite der Spanischen Republik. Hrsg. vom Dokumentationsarchiv des österreichischen Widerstandes. Wien: Österreichischer Bundesverlag 1986.

Hackl, Erich: Auroras Anlaß. Erzählung. Zürich: Diogenes 1987.

Hackl, Erich: In fester Umarmung. Geschichten und Berichte. Zürich: Diogenes 1996.

Hackl, Erich: Entwurf einer Liebe auf den ersten Blick. Erzählung. Zürich: Diogenes 1999.

Hackl, Erich: Die Hochzeit von Auschwitz. Eine Begebenheit. Zürich: Diogenes 2002.

Hackl, Erich, Cristina Timón Solinís (Hrsg.): $G e-$ schichten aus der Geschichte des Spanischen Bürgerkriegs. Erzählungen und Berichte deutschsprachiger Autoren. Darmstadt, Neuwied: Luchterhand 1986. Kantorowicz, Alfred (Hrsg.): Tschapajew. Das Bataillon der 21 Nationen. Barcelona, Madrid: Diana 1938.

Kantorowicz, Alfred: Spanisches Kriegstagebuch. Frankfurt/Main: Fischer 1982. (EA 1948)

Kesten, Hermann: Die Kinder von Gernika. Roman. Frankfurt/Main et al.: Ullstein 1981. (EA 1939)

Kirsch, Hans-Christian (Hrsg.): Der Spanische Bürgerkrieg in Augenzeugenberichten. Düsseldorf: Rauch 1967.

Koestler, Arthur: Ein spanisches Testament. Frankfurt/Main: Fischer 1980. (EA Spanish Testament 1937, deutsche EA 1938)

Landauer, Hans, Erich Hackl: Lexikon der österreichischen Spanienkämpfer 1936-1939. Wien: Theodor Kramer Gesellschaft 2003. Spanische Fassung: Diccionario de los voluntarios austriacos en la España republicana 1936-1939. Übers. von Bettina Linares Pérez. Madrid: Asociación de los Amigos de las Brigadas Internacionales 2005

Merin, Peter: Spanien zwischen Tod und Geburt. Zürich: Jean Christophe 1937.

Otten, Karl: Torquemadas Schatten. Frankfurt/Main: Fischer 1982. (EA 1938.)

Pichler, Georg: Der spanische Bürgerkrieg (19361939) im deutschsprachigen Roman. Eine Darstellung. Frankfurt/Main et al.: Lang 1991.

Regler, Gustav: Das Obr des Malchus. Eine Lebensgeschichte. Köln, Berlin: Kiepenheuer \& Witsch 1958.

Regler, Gustav: Juanita. Roman aus dem Spanischen Bürgerkrieg. Frankfurt/Main et al.: Büchergilde Gutenberg 1986.

Regler, Gustav: Der große Kreuzzug. Basel, Frankfurt/Main: Stroemfeld 1996. (EA The Great Crusade 1940; deutsche EA 1976)

Renn, Ludwig: Der spanische Krieg. Berlin: Aufbau 1955.

Spiess, Heiner (Hrsg.): «... dass Friede und Glück Europas vom Sieg der spanischen Republik abbängt.» Schweizer im Spanischen Bürgerkrieg. Zürich: Limmat 1986.

Thelen, Albert Vigoleis: Die Insel des zweiten Gesichts. Aus den angewandten Erinnerungen des Vigoleis. Frankfurt/Main, Berlin: Ullstein 1987. (EA 1953) Uhse, Bodo: Leutnant Bertram. Roman. Berlin, Weimar: Aufbau 1974. (EA 1943)

Weiss, Peter: Die Ästhetik des Widerstands. Roman. Frankfurt/Main: Suhrkamp 1975-1981.

\section{Anmerkungen}

1. Eine der wenigen Ausnahmen ist die spanische Übersetzung des von Hans Landauer und Erich Hackl erstellten Diccionario de los voluntarios austriacos en la España republicana 1936-1939. Übers. von Bettina Linares Pérez. Madrid: Asociación de los Amigos de las Brigadas Internacionales 2005.

2. Joseph Goebbels: Die Wabrheit über Spanien. Rede aufdem Reichsparteitag in Nürnberg 1937. München: Eher 1937, S. 3.

3. Willibrord Menke: Das Heldenlied vom Alkazar. Paderborn: Schöningh 1937, S. 47.

4. Alfred Kantorowicz:Spanisches Kriegstagebuch. Frankfurt/Main: Fischer 1982, S. 293.

5. Ludwig Renn: Der spanische Krieg. Berlin: Aufbau 1955, S. 42 bzw. 228.

6. Vgl. Kantorowicz, a.a.O., S. 292.

7. So etwa in der DDR Walter Gorrish in Um Spaniens Freiheit (1946) und Hanns Maassen in Die Söhne des Tschapajew (1960); im Westen erschienen die Romane von Gregor Stein Europäische Suite (1951), Rudolf Caltofen Juanita (1952) und Frederik Hetmann Bring den Schnee durchs Fener (1962). 